\title{
SOME THEOREMS ON ABSTRACT SPACES
}

\author{
BY R. E. A. C. PALEY*
}

1. Introduction. We shall solve some of the questions which are left unanswered by Banach in his treatise Théorie des Opérations Linéaires (Warszawa, 1932). They are taken from Chapter 12, pp. 193-207, and we shall assume throughout this paper that the reader is familiar with the ideas and results of that section.

We denote by $l^{(k)}$ the set of sequences $\left\{a_{1}, a_{2}, \ldots\right\}$ such that $\sum\left|a_{n}\right|^{k}<\infty$, and we denote by $L^{(k)}$ the set of measurable functions $f(x)$ defined on the interval $(0,1)$ for which

$$
\int_{0}^{1}|f(x)|^{k} d x<\infty .
$$

Suppose that $A$ and $B$ are two vectorial spaces. We say that $\operatorname{dim}_{l}(A) \geqq \operatorname{dim}_{l}(B)$, if $B$ is isomorphic with a vectorial subspace of $A$, and that $\operatorname{dim}_{l}(A)=\operatorname{dim}_{l}(B)$, if $\operatorname{dim}_{l}(A) \geqq \operatorname{dim}_{l}(B)$ and $\operatorname{dim}_{l}(B) \geqq \operatorname{dim}_{l}(A)$. Thirdly, we say that $\operatorname{dim}_{l}(A)>\operatorname{dim}_{l}(B)$, if $\operatorname{dim}_{l}(A) \geqq \operatorname{dim}_{l}(B)$ and if it is not true that $\operatorname{dim}_{l}(A)=\operatorname{dim}_{l}(B)$.

Banach has shown (loc. cit.) that if $\operatorname{dim}_{l}\left(l^{(q)}\right) \geqq \operatorname{dim}_{l}\left(L^{(p)}\right)$ for $q>1<p$, then $q=p=2$, and that $\operatorname{dim}_{l}\left(L^{(p)}\right) \geqq \operatorname{dim}_{l}\left(l^{(p)}\right)$, $p>1$, where the equality sign occurs only when $p=2$. He has also shown that the relation $\operatorname{dim}_{l}\left(l^{(q)}\right) \leqq \operatorname{dim}_{l}\left(L^{(p)}\right)$ is impossible if $q>p>2$ and $q<p<2$. We shall show that this relation is also impossible in the cases $\dagger$ (i) $p>2>q$, (ii) $q>2>p$, (iii) $2<q<p$.

2. Certain Inequalities. Let us suppose that $\operatorname{dim}_{l}\left(l^{(q)}\right)$ $\leqq \operatorname{dim}_{l}\left(L^{(p)}\right),(q>1<p)$. Then $l^{(q)}$ is isomorphic with a vectorial closed subspace of $L^{(p)}$. Suppose that to the sequences, $(1,0,0, \cdots),(0,1,0, \cdots),(0,0,1,0, \cdots), \cdots$ of $l^{(q)}$ correspond functions, $f_{1}(x), f_{2}(x), f_{3}(x), \cdots$ of $L^{(p)}$. Then to the sequence $\left(a_{1}, a_{2}, \cdots, a_{r}, 0,0, \cdots\right)$ corresponds

* This note was found, in rather incomplete form, among the papers of the late R. E. A. C. Paley. Various modifications and corrections were needed in order to bring it to its present form, but an effort was made to keep as close to the original as possible. F. J. Murray.

$\dagger$ We were not able to reconstruct the proof in the remaining case $p<q<2$, from the fragments contained in the manuscript. F.J.M. 
the function $a_{1} f_{1}(x)+a_{2} f_{2}(x)+\cdots+a_{r} f_{r}(x)$. Since the two spaces are isomorphic, it follows that the operation and its inverse are both limited and thus (see Banach, loc. cit., Theorem I, p. 54) the ratio

$$
\left\{\int_{0}^{1}\left|\sum_{m=1}^{n} a_{m} f_{m}(x)\right|^{p} d x\right\}^{1 / p} /\left\{\sum_{m=1}^{n}\left|a_{m}\right|^{q}\right\}^{1 / q}
$$

must lie between two positive constants.

Let $\phi_{1}(t), \phi_{2}(t), \cdots$ denote Rademacher functions.* We use the following inequality due to Khintchine. $\dagger$ We have, for $k>0$,

(1) $B_{k} \leqq\left(\int_{0}^{1}\left|\sum a_{n} \phi_{n}(t)\right|^{k} d t\right) /\left(\sum\left|a_{n}\right|^{2}\right)^{k / 2} \leqq B_{k}$;

here and in the sequel $B$ denotes a positive absolute constant, $B_{k}$ denotes one which depends only on $k$, and so on $\left(B, B_{k}, \cdots\right.$ may denote different constants in different contexts).

Now suppose that

$$
B_{p, q} \leqq\left\{\int_{0}^{1}\left|\sum a_{n} f_{n}(x)\right|^{p} d x\right\}^{1 / p} /\left\{\sum\left|a_{n}\right|^{q}\right\}^{1 / q} \leqq B_{p, q} .
$$

Then we have, for every $t$,

$$
B_{p, q} \leqq\left\{\int_{0}^{1}\left|\sum a_{n} \phi_{n}(t) f_{n}(x)\right|^{p} d x\right\}^{1 / p} /\left\{\sum\left|a_{n}\right|^{q}\right\}^{1 / q} \leqq B_{p, q},
$$

and thus raising to the $p$ th power, integrating with respect to $t$, using (1), and then extracting the $p$ th root, we find

$$
B_{p, q} \leqq \frac{\left[\int_{0}^{1}\left\{\sum\left|a_{n}\right|^{2}\left|f_{n}(x)\right|^{2}\right\}^{p / 2} d x\right]^{1 / p}}{\left\{\sum\left|a_{n}\right|^{q}\right\}^{1 / q}} \leqq B_{p, q} .
$$

We observe that if we take $a_{m}=1, a_{n}=0, n \neq m$, then (2) shows that

* See Rademacher 4, Khintchine 2, Paley and Zygmund 3, Kaczmarz and Steinhaus 1.

$\dagger$ Khintchine 2, Paley and Zygmund 3. 


$$
B_{p, q} \leqq\left[\int_{0}^{1}\left|f_{m}(x)\right|^{p} d x\right]^{1 / p} \leqq B_{p, q} .
$$

3. Proof for Cases (i) and (ii). First suppose that $p>2>q$. Then

$$
\begin{aligned}
\left\{\sum\left|a_{n}\right|^{q}\right\}^{1 / q} & \leqq B_{p, q}\left[\int_{0}^{1}\left\{\sum\left|a_{n}\right|^{2}\left|f_{n}(x)\right|^{2}\right\}^{p / 2} d x\right]^{1 / p} \\
& =B_{p, q}\left(\left[\int_{0}^{1}\left\{\sum\left|a_{n}\right|^{2}\left|f_{n}(x)\right|^{2}\right\}^{p / 2} d x\right]^{2 / p}\right)^{1 / 2} \\
& \leqq B_{p, q}\left(\sum\left[\int_{0}^{1}\left\{\left|a_{n}\right|^{2}\left|f_{n}(x)\right|^{2}\right\}^{p / 2} d x\right]^{2 / p}\right)^{1 / 2} \\
& =B_{p, q}\left(\sum\left|a_{n}\right|^{2}\left[\int_{0}^{1}\left|f_{n}(x)\right|^{p} d x\right]^{2 / p}\right)^{1 / 2} \\
& \leqq B_{p, q}\left[\sum\left|a_{n}\right|^{2}\right]^{1 / 2}
\end{aligned}
$$

which is of course impossible.

Suppose that $q>2>p$. Then

$$
\begin{gathered}
\left\{\sum\left|a_{n}\right|^{q}\right\}^{1 / q} \geqq B_{p, q}\left[\int_{0}^{1}\left\{\sum\left|a_{n}\right|^{2}\left|f_{n}(x)\right|^{2}\right\}^{p / 2} d x\right]^{1 / p} \\
=B_{p, q}\left[\left(\sum_{m}\left|a_{m}\right|^{2}\right)^{p / 2}\right. \\
\left.\cdot \int_{0}^{1}\left\{\sum_{n}\left(\left|a_{n}\right|^{2} /\left(\sum_{m}\left|a_{m}\right|^{2}\right)\right)\left|f_{n}(x)\right|^{2}\right\}^{p / 2} d x\right]^{1 / p} \\
\geqq B_{p, q}\left[\left(\sum_{m}\left|a_{m}\right|^{2}\right)^{p / 2} \sum_{n}\left(\left|a_{n}\right|^{2} /\left(\sum_{m}\left|a_{m}\right|^{2}\right) \int_{0}^{1}\left|f_{n}(x)\right|^{p} d x\right)\right]^{1 / p} \\
\geqq B_{p, q}\left(\sum\left|a_{n}\right|^{2}\right)^{1 / 2} .
\end{gathered}
$$

This too is impossible.

4. Proof for Case (iii). Suppose that $2<q<p$. Let

$$
\sum_{n=2}^{\infty}\left|a_{n}\right|^{q}=A^{q}
$$

Then 


$$
\begin{aligned}
& \int_{0}^{1}\left|f_{1}(x)\right|^{p-2}\left(\sum_{2}^{\infty}\left|a_{n}\right|^{2}\left|f_{n}(x)\right|^{2}\right) d x \\
& =A^{2-p} \int_{0}^{1}\left(A^{2}\left|f_{1}(x)\right|^{2}\right)^{p / 2-1}\left(\sum_{2}^{\infty}\left|a_{n}\right|^{2}\left|f_{n}(x)\right|^{2}\right) d x \\
& \leqq A^{2-p} \int_{0}^{1}\left\{A^{2}\left|f_{1}(x)\right|^{2}+\sum_{2}^{\infty}\left|a_{n}\right|^{2}\left|f_{n}(x)\right|^{2}\right\}^{p / 2} d x \leqq B_{p, q} A^{2}
\end{aligned}
$$

Thus we have

$$
\sum_{2}^{\infty}\left|a_{n}\right|^{2} \int_{0}^{1}\left|f_{1}(x)\right|^{p-2}\left|f_{n}(x)\right|^{2} d x \leqq B_{p, q}\left(\sum_{2}^{\infty}\left|a_{n}\right|^{q}\right)^{2 / q},
$$

and so it follows that

$$
\left.\int_{0}^{1}\left|f_{1}(x)^{p-2}\right| f_{n}(x)\right|^{2} d x \rightarrow 0 \text { as } n \rightarrow \infty .
$$

Let $N$ be a large finite number. Choose $n_{1}=1, n_{2}, n_{3}, \cdots, n_{N}$, so that

$$
\begin{array}{ll}
\int_{0}^{1}\left|f_{1}(x)\right|^{p-2}\left|f_{n_{k}}(x)\right|^{2} d x \leqq e^{-N}, & (k=2,3, \cdots, N), \\
\int_{0}^{1}\left|f_{n_{2}}(x)\right|^{p-2}\left|f_{n_{k}}(x)\right|^{2} d x \leqq e^{-N}, & (k=3,4, \cdots, N),
\end{array}
$$

and so on. We set $f_{n_{k}}(x)=g_{k}(x)$ and consider the integral

$$
\int_{0}^{1}\left(\sum_{k=1}^{N}\left|f_{n_{k}}(x)\right|^{2}\right)^{p / 2} d x=\int_{0}^{1}\left(\sum_{k=1}^{N}\left|g_{k}(x)\right|^{2}\right)^{p / 2} d x .
$$

Let $\mu$ be the integer such that $\mu \leqq p / 2<\mu+1$. Then the last integral does not exceed

$$
\begin{aligned}
\sum_{k_{1}=1}^{N} & \sum_{k_{2}=1}^{N} \ldots \sum_{k_{\mu+1}=1}^{N} \int_{0}^{1}\left|g_{k_{1}}(x)\right|^{2}\left|g_{k_{2}}(x)\right|^{2} \cdots\left|g_{k_{\mu+1}}(x)\right|^{p-2 \mu} d x \\
& \leqq \sum_{k=1}^{N} \int_{0}^{1}\left|g_{k}(x)\right|^{p} d x \\
& +N^{\mu+1} \max _{k_{1}, \ldots, k_{\mu+1} \text { not all equal }} \int_{0}^{1}\left|g_{k_{1}}(x)\right|^{2} \cdots\left|g_{k_{\mu+1}}(x)\right|^{p-2 \mu} d x .
\end{aligned}
$$


Now

$$
\begin{aligned}
& \int_{0}^{1}\left|g_{k_{1}}(x)\right|^{2} \cdots\left|g_{k_{\mu+1}}(x)\right|^{p-2 \mu} d x \\
& \leqq\left\{\int_{0}^{1}\left|g_{k_{1}}(x)\right|^{2}\left|g_{k_{2}}(x)\right|^{p-2} d x\right\}^{2 /(p-2)} \\
& \cdot\left\{\int_{0}^{1}\left|g_{k_{1}}(x)\right|^{2}\left|g_{k_{3}}(x)\right|^{p-2} d x\right\}^{2 /(p-2)} \ldots \\
& \cdot\left\{\int_{0}^{1}\left|g_{k_{1}}(x)\right|^{2}\left|g_{k_{\mu+1}}(x)\right|^{p-2} d x\right\}^{(p-2 \mu) /(p-2)},
\end{aligned}
$$

and if $k_{1}, k_{2}, \cdots, k_{\mu+1}$ are not all equal, $k_{1}$ must be different from one of the $k_{2}, k_{3}, \cdots, k_{\mu+1}$, and since each of the integrals in the above product is certainly bounded by a constant of the form $B_{p, q}$, it follows that the last expression does not exceed an expression of the form

$$
B_{p, q}\left\{\int_{0}^{1}\left|g_{k}(x)\right|^{2}\left|g_{k^{\prime}}(x)\right|^{p-2} d x\right\}^{(p-2 \mu) /(p-2)},
$$

where $k \neq k^{\prime}$. If now $k<k^{\prime}$, then

$$
\int_{0}^{1}\left|g_{k}(x)\right|^{2}\left|g_{k^{\prime}}(x)\right|^{p-2} d x \leqq e^{-N},
$$

while if $k>k^{\prime}, p \geqq 4$,

$$
\begin{aligned}
& \int_{0}^{1}\left|g_{k}(x)\right|^{2}\left|g_{k^{\prime}}(x)\right|^{p-2} d x \\
& \leqq\left\{\int_{0}^{1}\left|g_{k}(x)\right|^{p-2}\left|g_{k^{\prime}}(x)\right|^{2} d x\right\}^{2 /(p-2)}\left\{\int_{0}^{1}\left|g_{k^{\prime}}(x)\right|^{p} d x\right\}^{(p-4) /(p-2)} \\
& \leqq B_{p, q} e^{-2 N /(p-2)},
\end{aligned}
$$

and if $p \leqq 4$,

$$
\begin{aligned}
& \int_{0}^{1}\left|g_{k}(x)\right|^{2}\left|g_{k^{\prime}}(x)\right|^{p-2} d x \\
& \leqq\left\{\int_{0}^{1}\left|g_{k}(x)\right|^{p-2}\left|g_{k^{\prime}}(x)\right|^{2} d x\right\}^{(p-2) / 2}\left\{\int_{0}^{1}\left|g_{k}(x)\right|^{p} d x\right\}^{(4-p) / 2} \\
& \leqq B_{p, q} e^{-(p-2) N / 2}
\end{aligned}
$$


Hence the expression (3) is less than an expression of the form

$B_{p, q}\left\{N+N^{\mu+1}\left(e^{-N}+e^{-2 N /(p-2)}+e^{-(p-2) N / 2)^{(p-2 \mu) /(p-2)}}\right\} \leqq B_{p, q} N\right.$, if $N$ is chosen sufficiently large. This contradicts the hypothesis

$$
\int_{0}^{1}\left\{\sum_{k=1}^{N}\left|f_{n_{k}}(x)\right|^{2}\right\}^{p / 2} d x \geqq B_{p, q} N^{p / q},
$$

and we have shown that (iii) is impossible.

5. Conclusion. In conclusion it might be pointed out that if the relation $\operatorname{dim}_{l}\left(l^{(q)}\right) \leqq \operatorname{dim}_{l}\left(L^{(p)}\right)$ is impossible, then also the relation $\operatorname{dim}_{l}\left(L^{(q)}\right) \leqq \operatorname{dim}_{l}\left(L^{(p)}\right)$ is impossible. For if $\operatorname{dim}_{l}\left(L^{(q)}\right) \leqq \operatorname{dim}_{l}\left(L^{(p)}\right)$, then since $\operatorname{dim}_{l}\left(l^{(q)}\right)<\operatorname{dim}_{l}\left(L^{(q)}\right)$, it follows that $\operatorname{dim}_{l}\left(l^{(q)}\right) \leqq \operatorname{dim}_{l}\left(L^{(p)}\right)$, a contradiction. Thus in each of the Cases (i), (ii), and (iii), we also have the statement of the impossibility of the relationship $\operatorname{dim}_{l}\left(L^{(q)}\right) \leqq \operatorname{dim}_{l}\left(L^{(p)}\right)$. For Cases (i) and (ii), these last results are given by Banach (loc. cit.); however Case (iii) is new.

\section{REFERENCES}

1. S. Kaczmarz and H. Steinhaus, Le système orthogonal de M. Rademacher, Studia Mathematica, vol. 2 (1930), pp. 231-247.

2. A. Khintchine, Über dyadische Brüche, Mathematische Zeitschrift, vol. 18 (1923), pp. 109-116.

3. R. E. A. C. Paley and A. Zygmund, On some series of functions, Proceedings of the Cambridge Philosophical Society, vol. 26 (1930), pp. 337-357.

4. H. Rademacher, Einige Sätze über Reihen von allgemeine Orthogonalfunktionen, Mathematische Annalen, vol. 87 (1922), pp. 112-138. 